\title{
Distributed Weight-Average-Prediction Control and Stability Analysis for an Islanded Microgrid with Communication Time Delay
}

Yao, Weitao; Wang, Yu; Xu, Yan; Deng, Chao; Wu, Qiuwei

Published in:

IEEE Transactions on Power Systems

Link to article, DOI:

10.1109/TPWRS.2021.3092717

Publication date:

2021

Document Version

Peer reviewed version

Link back to DTU Orbit

Citation (APA):

Yao, W., Wang, Y., Xu, Y., Deng, C., \& Wu, Q. (2021). Distributed Weight-Average-Prediction Control and Stability Analysis for an Islanded Microgrid with Communication Time Delay. IEEE Transactions on Power Systems, 37(1), 330 - 342. https://doi.org/10.1109/TPWRS.2021.3092717

\section{General rights}

Copyright and moral rights for the publications made accessible in the public portal are retained by the authors and/or other copyright owners and it is a condition of accessing publications that users recognise and abide by the legal requirements associated with these rights.

- Users may download and print one copy of any publication from the public portal for the purpose of private study or research.

- You may not further distribute the material or use it for any profit-making activity or commercial gain

- You may freely distribute the URL identifying the publication in the public portal 


\title{
Distributed Weight-Average-Prediction Control and Stability Analysis for an Islanded Microgrid with Communication Time Delay
}

\author{
Weitao Yao, Student Member, IEEE, Yu Wang, Member, IEEE, Yan Xu, Senior Member, IEEE, Chao \\ Deng, Member, IEEE, and Qiuwei Wu, Senior Member, IEEE
}

\begin{abstract}
This paper studies the communication time-delay issues in islanded microgrids (MGs) with the distributed secondary control architecture. Firstly, a time-delayed MG smallsignal model is developed. Then, a new weight-average-prediction (WAP) controller is proposed to compensate the delayed system state. By introducing a time-delayed differential term in the proposed control law, the traditional time-delayed small-signal model is transformed into a neutral time-delayed mathematic model. Based on the developed model, the stability analysis is conducted considering both fixed time delay and time-varying delay. For the fixed time delay, a novel graphic analytical method is proposed to evaluate the time delay margin, which eliminates the conservatism compared with existing time-domain methods. For the time-varying delay, stability condition is established by a Lyapunov-Krasovskii function and linear matrix inequalities. In addition, some non-linear WAP control methods are discussed to guide the parameter tuning with a higher resolution. Lastly, the proposed method and analytical result are verified in the OPALRT real-time test platform. The results demonstrate the effectiveness and high performance of the proposed controller.
\end{abstract}

Index Terms - Communication time delay, distributed control, microgrid, small-signal model, weight-average-prediction.

\section{INTRODUCTION}

$\mathrm{M}$ icrogrids (MGs) are becoming vital parts of the future smart grid with the higher penetration of renewable energy sources [1]. Compared with the traditional power system, MGs have the advantages such as being eco-friendly, flexible and can enhance grid resilience. Typically, MGs can operate in either grid-connected or islanded mode [2]. In the islanded mode, to ensure stable operation and optimal dispatch in MGs, a typical hierarchical control framework including primary, secondary and tertiary control is implemented [3]. The droopbased primary control ensures the MG system stability by a rapid response of local controllers. The secondary control aims to eliminate the frequency and voltage deviation caused by primary control. The tertiary control is related to the economic dispatch. In this paper, we focus on the secondary control layer of islanded MG systems, where the communication time-delay issue is to be investigated.

Generally, the secondary control can be achieved in a

The work in this paper was supported in part by Ministry of Education (MOE), Republic of Singapore, under grant AcRF TIER 1 2019-T1-001-069 (RG75/19). Corresponding author: Yan Xu. (e-mail: xuyan@ntu.edu.sg)

W. Yao is with ERI@N, Interdisciplinary Graduate School, Nanyang Technological University, Singapore, 637141.

Y. Wang is with Rolls-Royce@NTU Corporate Lab, Nanyang Technological University, Singapore, Nanyang 50 Ave, 639798. centralized way or distributed way [4]. The centralized controller requires a fully connected communication network and hence suffers from higher single-point failure risk and heavier computation burden. By contrast, the distributed control is more reliable due to its independence on a central controller. Based on the consensus algorithms, various distributed secondary control methods have been proposed, such as finitetime controller [3], event-triggered controller [5] and model predictive control [6].

However, the communication time delay [7] in distributed control, as a practical issue, has not been addressed by the above works. Typically, the time delay on an MG network includes the transmission delay, propagation delay, queuing delay, and routing delay, which adds latency into the network and may lead to system instability [8]. Therefore, how to determine the delay-dependent stability conditions and enhance the time delay margin (TDM) for the time-delayed MG system are of great importance [9].

For existing works, there are two types of approaches to determine the delay-dependent stability conditions: frequencydomain approach [10]-[13] and time-domain approach [14][19]. The frequency-domain methods focus on the evaluation of the eigenvalues by solving the closed-loop system characteristic equation [20]. By searching the critical point in the imaginary axis, the critical stability condition can be obtained [11]-[12]. However, the frequency-domain methods have a drawback of high computation burden and they cannot deal with the timevarying delay scenario and complex non-linear system models. To solve this issue, the time-domain methods are proposed to define a sufficient stability condition, which allows dealing with system uncertainties and stochastic disturbance [18]-[19]. However, conservativeness exists in time-domain methods, which limits the design of control parameters. In this paper, to address the above issues, a novel graphic analytical method based on the generalized Nyquist theorem is proposed to evaluate the fixed TDM for the time-delayed MG system. Besides, for the time-varying delay scenario, the stability condition is established by the Lyapunov-Krasovskii function and linear matrix inequalities (LMIs).

Another critical issue to be addressed is to enhance the TDM

Y. Xu and C. Deng are with School of Electrical and Electronic Engineering, Nanyang Technological University, Singapore, Nanyang 50 Ave, 639798.

Q. Wu is with Department of Electrical Engineering, Technical University of Denmark, Lyngby, Denmark. 
TABLE I COMPARISON OF EXISTING WORKS

\begin{tabular}{|c|c|c|c|c|c|c|c|c|}
\hline \multirow[b]{2}{*}{ Reference } & \multicolumn{4}{|c|}{ Control Method } & \multicolumn{4}{|c|}{ Stability Analysis } \\
\hline & $\begin{array}{c}\text { Gain- } \\
\text { scheduling } \\
\text { based }\end{array}$ & $\begin{array}{c}\text { Prediction } \\
\text { /Compensation } \\
\text { based }\end{array}$ & Distributed & Centralized & $\begin{array}{l}\text { Frequency- } \\
\text { domain } \\
\text { method }\end{array}$ & $\begin{array}{l}\text { Time- } \\
\text { domain } \\
\text { method }\end{array}$ & $\begin{array}{l}\text { Fixed } \\
\text { delay }\end{array}$ & $\begin{array}{c}\text { Time- } \\
\text { varying } \\
\text { delay }\end{array}$ \\
\hline$[10,26]$ & $\sqrt{ }$ & & & $\sqrt{ }$ & $\sqrt{ }$ & & $\sqrt{ }$ & \\
\hline [20] & & $\sqrt{ }$ & $\sqrt{ }$ & & $\sqrt{ }$ & & $\sqrt{ }$ & \\
\hline [27] & $\checkmark$ & & & $\sqrt{ }$ & & $\sqrt{ }$ & $\sqrt{ }$ & \\
\hline [28] & & $\sqrt{ }$ & $\sqrt{ }$ & & $\sqrt{ }$ & & $\sqrt{ }$ & \\
\hline This paper & & $\sqrt{ }$ & $\sqrt{ }$ & & $\sqrt{ }$ & $\sqrt{ }$ & $\sqrt{ }$ & $\sqrt{ }$ \\
\hline
\end{tabular}

for time-delayed MG system. Up to now, most of the existing works focus on the controller design and determination of TDM, and very few works are reported for the TDM enhancement. For example, reference [16] defines the TDM considering a switching communication network and different topology connectivity. However, it only focuses on $\mathrm{f} / \mathrm{V}$ control and only a delay-dependent stability condition is given. Reference [15] and [21] propose the distributed resilient controllers by applying $\mathrm{H} \infty$ theory. Reference [22] studies aperiodic sampled time-delay issue in MG secondary control and reference [23] proposes an optimal design for a directed communication topology and pinning location. However, these references only give the upper bound of time delay and no TDM enhancement strategy is reported. The model predictive controller and Smith predictor in the MG control layer are proposed in [24] to improve system performance with communication delay, but only stability conditions are determined. Similarly, eventtriggered control [25] has also been investigated but it also only focuses on controller design.

For the limited existing TDM-enhancement works, there are mainly two types of approaches to improve the TDM: gainscheduling-based method and prediction/compensation-based method. For example, references [10] and [26] propose a gainscheduling method to improve TDM by dynamically adjusting the control gain but it sacrifices the system convergence speed due to the decreased control gains. The delay issue in shipboard MGs is studied and an LMI-based controller is proposed in [27] against the delay. However, the method is also based on the gain-scheduling principle and the adaptive gain is calculated by LMIs. Besides, the proposed controller in [27] is centralized and the time-varying delay is not considered. By comparison, prediction/compensation-based method shows a better system performance without the sacrifice of the system convergence speed. The delay compensators based on lead-lag compensation are proposed in [20] and [28] but the method is only suitable for the time-delayed MG with fixed time delay.

To fill the above gaps, this paper proposes a distributed weight-average-prediction (WAP) controller to enhance the TDM for the time-delayed MG system. Firstly, a time-delayed MG small-signal model is developed. Then, a WAP-based control method is proposed based on the compensation of the system state. Besides, by introducing a time-delayed differential term in the proposed control law, the traditional time-delayed small-signal model is transformed to a neutral time-delayed mathematic model. Based on the developed model, the stability analysis is conducted considering both fixed time delay and time-varying delay scenarios. A real-time experimental simulation platform based on OPAL-RT is used to verify the performance of the proposed controller and stability conditions. In summary, the salient contributions of this paper are listed as follows.

1) A new distributed WAP controller is designed to improve the tolerance of MG system to time delay. Different from most existing methods which only determine the upper bound of TDM, the proposed WAP controller aims to enhance the TDM for the time-delayed MG system.

2) The stability analysis is conducted based on the transformed neutral time-delayed mathematic model. Both the fixed delay and time-varying delay scenarios are analyzed. For fixed time delay, a novel graphic analytical method is proposed. As a frequency-domain analytical method, it eliminates the conservatism of existing time-domain analytical methods and reduces the computational burden. Besides, the stability condition for the time-varying delay is investigated by a Lyapunov-Krasovskii function and LMIs.

3) Three non-linear predictor coefficient functions are discussed to guide the parameter tuning with a higher resolution.

A general comparison in the control method and stability analysis between existing literature and this paper is made in Table I.

\section{Mathematical Modeling}

\section{A. Small-Signal Model of MG System}

Generally, the MG control system is designed in the $d-q$ reference frame. In terms of the angle deviation between the local frame and reference frame, the state variables in the MG system are transformed into the $d-q$ frame of reference inverter (inverter 1) based on the following technique,

$$
\left[f_{D Q}\right]=T_{i}\left[f_{d q}\right] \quad T_{i}=\left[\begin{array}{lr}
\cos \left(\delta_{i}-\delta_{1}\right) & -\sin \left(\delta_{i}-\delta_{1}\right) \\
\sin \left(\delta_{i}-\delta_{1}\right) & \cos \left(\delta_{i}-\delta_{1}\right)
\end{array}\right]
$$

where $f_{D Q}=\left[f_{D} f_{Q}\right]^{T}$ and $f_{d q}=\left[f_{d} f_{q}\right]^{T} . \delta_{i}$ is the angle of the local $d q$-frame of each inverter.

DER unit: The control structure of each DER includes primary control and secondary control, as shown in Fig. 1. Generally, there are three control loops, namely, the voltage, current and droop control loops, in the primary controller. In this paper, the dynamics of the voltage control loop, current control loop are neglected in the small-signal modeling, which is a mild assumption to study the network level dynamics [3], [11]. To decouple the f/P control and V/Q droop control with 


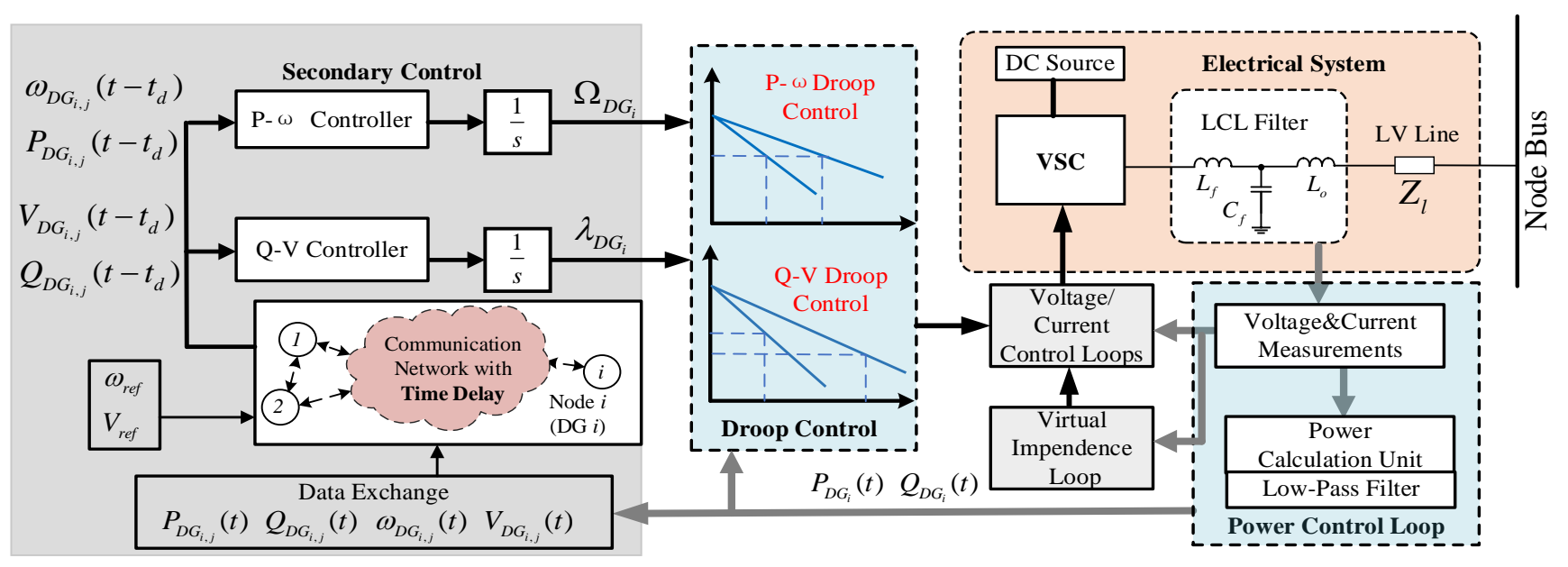

Fig. 1 The illustration of a typical hierarchical control framework

better system performance, the virtual impedance is introduced into the primary control and the detailed dynamic model is shown as follow [12],

$$
\left[\begin{array}{l}
v_{v d} \\
v_{v q}
\end{array}\right]=\left[\begin{array}{cc}
R_{v} & -\omega L_{v} \\
\omega L_{v} & R_{v}
\end{array}\right]\left[\begin{array}{l}
i_{o d} \\
i_{o q}
\end{array}\right]
$$

where $v_{v d}$ and $v_{v q}$ are the voltage drops over the virtual impedance $R_{v}$ and $L_{v}$ in $d-q$ frame. Hence, the output voltage of $i$ th DER in $d-q$ frame can be calculated from the droop reference voltage $v_{r d} / v_{r q}$ subtracting the virtual voltage drops as follows,

$$
\left\{\begin{array}{l}
v_{o d_{i}}=v_{r d_{i}}-v_{v d_{i}} \\
v_{o q_{i}}=v_{r q_{i}}-v_{v q_{i}}
\end{array}\right.
$$

Considering a low-pass filter, the instantaneous active and reactive power is calculated as follow:

$$
\begin{aligned}
& P_{i}=\frac{3}{2} * \frac{\omega_{c}}{s+\omega_{c}}\left(v_{o D_{i}} i_{o d_{i}}+v_{o Q_{i}} i_{o q_{i}}\right) \\
& Q_{i}=\frac{3}{2} * \frac{\omega_{c}}{s+\omega_{c}}\left(v_{o Q_{i}} i_{o d_{i}}-v_{o D_{i}} i_{o q_{i}}\right)
\end{aligned}
$$

where $\omega_{c}$ is the cut-off frequency of low-pass filters. Noted only the voltage need to transform into the common reference frame. Therefore, the expression of voltage in equation (4) is presented as $v_{O D_{i}}$ and $v_{o Q_{i}}$, while the current is still expressed as $i_{o d_{i}}$ and $i_{o q_{i}}$. The droop control of power inverters is realized in local controllers. The droop characteristic of primary control is given as follows [15],

$$
\begin{aligned}
& \omega_{i}=\omega_{n}-k_{p_{i}} P_{i}+\Omega_{i} ; \\
& v_{r d_{i}}=V_{n}-k_{q_{i}} Q_{i}+\lambda_{i} ; \\
& v_{r q_{i}}=0 ;
\end{aligned}
$$

where $\omega_{n}$ and $V_{n}$ are the nominal setpoint of system frequency and $d$-axis output voltage. $k_{p_{i}}$ and $k_{q_{i}}$ are droop gains of $i$ th DER. Considering the output line impendence, we have:

$$
\begin{aligned}
& \frac{d i_{o d_{i}}}{d t}=-\frac{R_{i}}{L_{i}} i_{o d_{i}}+\omega_{1} i_{o q_{i}}+\frac{1}{L_{i}} v_{o D_{i}}-\frac{1}{L_{i}} v_{D_{k}} \\
& \frac{d i_{o q_{i}}}{d t}=-\frac{R_{i}}{L_{i}} i_{o q_{i}}-\omega_{1} i_{o d_{i}}+\frac{1}{L_{i}} v_{o Q_{i}}-\frac{1}{L_{i}} v_{Q_{k}}
\end{aligned}
$$

where $R_{i}$ and $L_{i}$ are the output line resistance and inductance. $v_{D_{k}}$ and $v_{Q_{k}}$ represent the $d q$-frame $k$ th node voltage. Based on the above equations, one can obtain the state equation of DER unit,

$$
\begin{aligned}
{\left[\Delta \dot{X}_{i n v_{i}}\right] } & =A_{s_{i}}\left[\Delta X_{i n v_{i}}\right]+B_{s_{i}} \Delta \dot{\Omega}_{i}+C_{s_{i}} \Delta \Omega_{i}+D_{s_{i}} \Delta \dot{\lambda}_{i} \\
& +E_{s_{i}} \Delta \lambda_{1}+F_{s_{i}}\left[\Delta \delta_{1} \Delta \omega_{1}\right]^{T}+G_{s_{i}}\left[\Delta v_{D Q_{k}}\right]
\end{aligned}
$$

parameter matrices $A_{s_{i}}, B_{s_{i}}, C_{s_{i}}, D_{s_{i}}, E_{s_{i}} F_{s_{i}}$ and $G_{s_{i}}$ are parameter matrices. The state variables of $D E R_{i}$ are

$$
\Delta X_{\text {inv }_{i}}=\left[\Delta \delta_{i}, \Delta \omega_{i}, \Delta v_{r d_{i}}, \Delta i_{o d_{i}}, \Delta i_{o q_{i}}\right]
$$

Secondary Control: Distributed secondary control depends on the information exchange (e.g., $\omega, V, P, Q$ ) among DERs and the aim is to restore frequency/voltage and achieve power sharing. Based on the consensus algorithm, a typical distributed secondary control law is designed as follows,

$$
\begin{aligned}
\omega_{i}= & \omega_{n}-k_{p_{i}} P_{i}+\Omega_{i} ; v_{r d_{i}}=V_{n}-k_{q_{i}} Q_{i}+\lambda_{i} \\
\dot{\Omega}_{i}= & c_{\omega i} \sum_{j \in N_{i}} a_{i j}\left(\omega_{j}-\omega_{i}\right)+c_{\omega i} \theta_{i}\left(\omega_{r e f}-\omega_{i}\right) \\
& +c_{p i} \sum_{j \in N_{i}} a_{i j}\left(k_{p_{j}} P_{j}-k_{p_{i}} P_{i}\right) \\
\dot{\lambda}_{i} & =c_{v i} \sum_{j \in N_{i}} a_{i j}\left(v_{o d_{j}}-v_{o d_{i}}\right)+c_{v i} \theta_{i}\left(V_{r e f}-v_{o d_{i}}\right) \\
& +c_{q i} \sum_{j \in N_{i}} a_{i j}\left(k_{q_{j}} Q_{j}-k_{q_{i}} Q_{i}\right)
\end{aligned}
$$

where $c_{\omega i}, c_{p i}, c_{v i}$ and $c_{q i}$ are the positive control gains. $a_{i j}$ and $\theta_{i}$ are the elements of the weighted adjacency matrix of the $i$ th DER and pinning gain matrix of MG. $\omega_{\text {ref }}$ and $V_{r e f}$ are the frequency and voltage reference value.

Remark 1: Note that only a perfectly symmetric microgrid can reach voltage restoration and reactive power sharing simultaneously due to the trade-off caused by the unbalanced line impedances and loads. This paper focuses on stability issues and the priority is to recover the voltage. Hence, reactive power-sharing is not considered in this paper.

Electrical Network: Two main components including loads and lines are modeled in the electrical network. Firstly, without loss of generalization, we assume that only one equivalent resistance-inductance (RL) load is connected to each bus in this paper. It can be easily expanded to a more complex system with more loads. The dynamic equation of RL load connected to node $k$ is, 


$$
\begin{aligned}
& \frac{d i_{\text {loadD }}}{d t}=-\frac{R_{\text {load }}}{L_{\text {load }}} i_{\text {laodD }}+\omega_{1} i_{\text {laodQ }}+\frac{1}{L_{\text {load }}} v_{D_{k}} \\
& \frac{d i_{\text {loadQ }}}{d t}=-\frac{R_{\text {load }}}{L_{\text {load }}} i_{\text {laodQ }}-\omega_{1} i_{\text {laodD }}+\frac{1}{L_{\text {load }}} v_{Q_{k}}
\end{aligned}
$$

where $v_{D_{k}}$ and $v_{Q_{k}}$ are the voltage of node $k$ in $d q$-frame.

Similarly, for line $i$ between node $j$ and node $k$, the equations of line current also can be obtained. The line model can be written as,

$$
\begin{gathered}
\frac{d i_{\text {line } D_{i}}}{d t}=-\frac{R_{\text {linei }}}{L_{\text {linei }}} i_{\text {line } D_{i}}+\omega_{1} i_{\text {line } Q_{i}}+\frac{1}{L_{\text {linei }}} v_{D_{j}}-\frac{1}{L_{\text {linei }}} v_{D_{k}} \\
\frac{d i_{\text {line } Q_{i}}}{d t}=-\frac{R_{\text {linei }}}{L_{\text {linei }}} i_{\text {line } Q_{i}}-\omega_{1} i_{\text {line } D_{i}}+\frac{1}{L_{\text {linei }}} v_{Q_{j}}-\frac{1}{L_{\text {linei }}} v_{Q_{k}}
\end{gathered}
$$

Then, by combining equation (7), (10)-(15) and linearization, the small-signal model of MG system is derived as follows and the detailed coefficient matrices can refer the reference [11] and [29],

$$
\left[\Delta \dot{X}_{M G}\right]=A_{I N V}\left[\Delta X_{M G}\right]
$$

where the MG system state variable is defined as:

$$
\begin{aligned}
\Delta X_{M G}=\left[\Delta X_{i n v_{1}} \ldots \Delta X_{i n v_{n}} \Delta \Omega_{1} \ldots \Delta \Omega_{n} \Delta \lambda_{1} \ldots \Delta \lambda_{n} \ldots\right. \\
\left.\ldots \Delta i_{\text {lined }_{1}} \ldots \Delta i_{\text {lined }_{n}} \Delta i_{\text {loadd }_{1}} \ldots \Delta i_{\text {loaddq }_{n}}\right]^{T}
\end{aligned}
$$

\section{B. Communication Time Delay Model}

For the MG communication system, the data are transmitted in the form of packets. If packets are not received at the destination end, some packet-loss will cause a communication time delay in the signal. Typically, the communication time delay $t_{d}$ has the following parts,

$$
t_{d}=t_{s}+t_{b}+t_{p}+t_{r}
$$

where $t_{s}=P_{s} / D_{r}$ is the serial delay, $P_{s}$ is the size of the packet (bits/s) and $D_{r}$ is the data rate of the communication network. $t_{b}$ is between packet delay. $t_{p}=l / v$ is the propagation delay where $l$ is the length of the communication medium and $v=$ $0.6 c$ is the propagation velocity ( $c$ is the light velocity). $t_{r}$ is the routing delay, which can be determined by $\mathrm{M} / \mathrm{M} / 1$ queuing method [30].

For the above delay components, the main source of uncertainty lies in $t_{r}$ and $t_{s}$. Routing delay $t_{r}$ depends on the bandwidth of the communication infrastructure and serial delay $t_{s}$ is impacted by the size change of the packet. In this paper, we will analyze and compare the fixed time delay and the timevarying delay caused by the data transmission, respectively. Firstly, it is assumed the communication bandwidth and packet size are certain. In this case, the system delay is considered as a fixed delay. Besides, a practical scenario with the uncertain $t_{r}$ and $t_{s}$ is considered and thus the time-varying delay is further analyzed.

\section{Time-Delayed MG Small-Signal Model}

Considering the time delay $t_{d}$ affecting the information exchange in secondary controllers of DERs, the initial secondary control law is rewritten as follow,

$$
\begin{aligned}
& \omega_{i}(t)=\omega_{n}-k_{p_{i}} P_{i}(t)+\Omega_{i}(t) ; \quad v_{r d_{i}}(t)=V_{n}-k_{q_{i}} Q_{i}(t)+\lambda_{i}(t) \\
& \dot{\Omega}_{i}(t)=c_{\omega i} \sum_{j \in N_{i}} a_{i j}\left(\omega_{j}\left(t-t_{d}\right)-\omega_{i}\left(t-t_{d}\right)\right)+c_{\omega i} \theta_{i}\left(\omega_{r e f}-\omega_{i}\left(t-t_{d}\right)\right) \\
& +c_{p i} \sum_{j \in N_{i}} a_{i j}\left(k_{p_{j}} P_{j}\left(t-t_{d}\right)-k_{p_{i}} P_{i}\left(t-t_{d}\right)\right) \\
& \dot{\lambda}_{i}(t)=c_{v i} \sum_{j \in N_{i}} a_{i j}\left(v_{o d_{j}}\left(t-t_{d}\right)-v_{o d_{i}}\left(t-t_{d}\right)\right)+c_{v i} \theta_{i}\left(V_{r e f}-v_{o d_{i}}\left(t-t_{d}\right)\right) \\
& \quad+c_{q i} \sum_{j \in N_{i}} a_{i j}\left(k_{q_{j}} Q_{j}\left(t-t_{d}\right)-k_{q_{i}} Q_{i}\left(t-t_{d}\right)\right)
\end{aligned}
$$

Then, a complete time-delayed MG small-signal model can be formed. The dynamic of the time-delayed MG system can be described as follows,

$$
\left[\Delta X_{M G}^{\bullet}(t)\right]=A_{I N V_{1}}\left[\Delta X_{M G}(t)\right]+A_{I N V_{2}}\left[\Delta X_{M G}\left(t-t_{d}\right)\right]
$$

where the MG system state variables are the same with equation (17). Noted a delayed term in state equation (22) is introduced due to the presence of a delayed term in secondary control law.

Remark 2: Equation (22) is a particular case $(i=1)$ of the linear delay differential algebraic equations (DDAE)

$$
\dot{x}(t)=A_{0} x(t)+\sum_{i=1}^{n} A_{i} x\left(t-t_{d_{i}}\right)
$$

which describes the time-delayed MG system with a single delay. The characteristic equation for the system is

$$
\operatorname{det}\left(-s I+A_{0}+A_{1} e^{-s t_{d}}\right)=0
$$

(24) has infinite roots and existing studies such as [13] and [19] have widely investigated this issue.

\section{Distributed WeIGHT-AVERAGE-PREDICTION CONTROLLER DESIGN}

In this section, the distributed WAP secondary controllers are designed to mitigate the impact of time delay on frequency/voltage restoration and power-sharing. The WAP control structure is introduced and the implementation of WAP control in the time-delayed MG system is studied.

For the secondary control protocols (20) and (21), the state information with time delay $t_{d}$ is defined as $x\left(t-t_{d}\right)$. As shown in Fig. 2, at time $t$, the system state received by receiver is $x\left(t-t_{d}\right)$ due to the time delay $t_{d}$. To reduce the impact of communication time delay on system performance, we wish to use $x\left(t-t_{d}+\sigma\right), 0<\sigma<t_{d}$ instead of $x\left(t-t_{d}\right)$ in the protocol (14) and (15). In this case, the system state is closer to the state without time delay. However, $x\left(t-t_{d}+\sigma\right)$ is not available for feedback due to the presence of time delay. To overcome the unavailability, a predicted state for $x\left(t-t_{d}+\sigma\right)$, $0<\sigma<t_{d}$ is defined as $\hat{x}\left(t-t_{d}+\sigma\right)$ by using $x\left(t-t_{d}\right)$ given as follow,

$$
\hat{x}\left(t-t_{d}+\sigma\right)=x\left(t-t_{d}\right)+\sigma \dot{x}\left(t-t_{d}\right), \quad 0<\sigma<t_{d}
$$




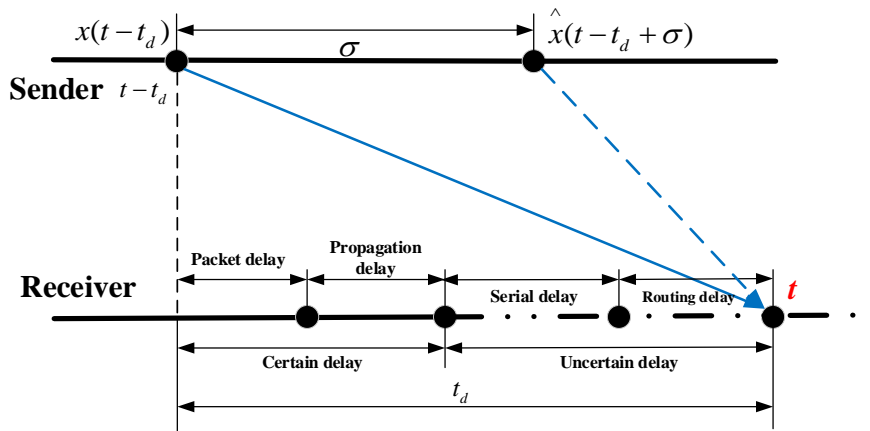

Fig. 2. General WAP control structure with certain time delay (packet delay and propagation delay) and uncertain delay (serial delay and routing delay).

where $\sigma$ is called the length of the weight average prediction.

Based on (25), the predicted states $\hat{x}$ in MG including frequency, voltage, active power and reactive power are listed

$$
\hat{x}=\left[\begin{array}{llll}
\hat{\omega} & \hat{v} & \hat{P} & \hat{Q}
\end{array}\right]^{T}
$$

Using $\hat{x}\left(t-t_{d}+\sigma\right)$ in (25) to replace $x\left(t-t_{d}\right)$ in (20) and (21), one can obtain the new WAP control laws as follow,

$$
\begin{aligned}
& \dot{\Omega}(t)=-c_{\omega} * L\left[\omega\left(t-t_{d}\right)+\sigma \dot{\omega}\left(t-t_{d}\right)\right]+c_{\omega} * G *\left(I * \omega_{\text {ref }}-\omega\left(t-t_{d}\right)\right) \\
&-c_{p} * L^{*} k_{p}\left[P\left(t-t_{d}\right)+\sigma \dot{P}\left(t-t_{d}\right)\right] \\
& \dot{\lambda}(t)=-c_{v} * L^{*}\left[v_{o d}\left(t-t_{d}\right)+\sigma \dot{v_{o d}}\left(t-t_{d}\right)\right]+c_{v} * G *\left(I * V_{\text {ref }}-v_{o d}\left(t-t_{d}\right)\right) \\
&-c_{q} * L^{*} k_{q}\left[Q\left(t-t_{d}\right)+\sigma \dot{Q}\left(t-t_{d}\right)\right]
\end{aligned}
$$

By combining the state equations of the primary controller, loads and network, the new time-delayed MG small-signal model is obtained,

$$
\Delta \dot{X}_{M G}(t)=A_{M G_{1}} \Delta X_{M G}(t)+A_{M G_{2}} \Delta X_{M G}\left(t-t_{d}\right)+A_{M G_{3}} \Delta \dot{X}_{M G}\left(t-t_{d}\right)
$$

Remark 3: This paper considers a uniform length $\sigma$ of the weight average prediction for different system states such as $\omega$, $V, P$ and $Q$. In practice, according to different state's sensitivity to communication time delay or the stability demand, the WAP length $\sigma$ can be individually designed.

Remark 4: Equation (29) belongs to the class of neutral timedelayed system,

$$
\dot{x}(t)-C \dot{x}\left(t-t_{d}\right)=A x(t)+A_{1} x\left(t-t_{d}\right)
$$

Compared with the previous time-delayed MG system (22), a time-delayed differential term is introduced in (29) due to the presence of a differential term in secondary control law, which transforms the DDAE problem into a stability problem of the neutral time-delayed system (NTDS).

Remark 5: The equilibrium point of the developed timedelayed MG small-signal model (29) exists when $\Delta \dot{X}_{M G}(t)-$ $A_{M G 3} \Delta \dot{X}_{M G}\left(t-t_{d}\right)=0$; When the system operates in the equilibrium point, by solving the equation $A_{M G 1} \Delta X_{M G}(t)+$ $A_{M G 2} \Delta X_{M G}\left(t-t_{d}\right)=0$, the system state in the equilibrium point can be determined. Note that the time-delayed differential term in (25) will not change the original equilibrium point due to the fixed system state matrix. The change of equilibrium point of the MG closed-loop system is only related to the system parameters changing or large system disturbance such as load change.

\section{StabiLITY ANALYSIS}

Based on the developed neutral time-delayed MG system, this section studies the small-signal stability considering fixed time delay and time-varying delay.

For stability analysis of traditional time-delayed MG system, the focus is on the solution of the DDAE characteristic equation and existing works have widely investigated this issue. However, stability analysis for NTDS such as (29) is difficult due to the introduction of the delayed differential term. Existing analytical methods mainly try to find a sufficient stability condition in time-domain but higher conservatism exists. In this paper, we propose a novel graphic analytical method to evaluate the fixed time delay. To our best knowledge, the proposed graphic method has not been applied to MG NTDS. Besides, for the complex time-varying delay, the time-domain method based on the Lyapunov-Krasovskii function and LMIs is introduced.

\section{A. Graphic Analytical Method for Fixed Time Delay Analysis}

As a graphical analytical method, the Nyquist stability criterion is widely used to evaluate the system dynamic stability especially for high-order or nonlinear systems [31]. For a MultiInput-Multi-Output (MIMO) system like the MG system, the generalized Nyquist stability criterion provides a useful tool to directly utilize the frequency-domain system state-space realizations. The following theorem is proposed with Fig. 3 to illustrate the generalized Nyquist stability criterion.

Theorem 1: Consider the MIMO feedback system shown in Fig. 3 where $G(s)$ and $K(s)$ are the transfer function matrix and $r$ and $y$ are input and output vectors. Supposing that there are no hidden modes (unstable pole-zero cancellations) and the total number of unstable poles of $G(s)$ and $K(s)$ is $X$, then the system will be close-loop stable if and only if the eigenloci of the return ratio $L(s)=G(s) K(s)$ encircles the point $(-1+j 0)$ $X$ times in a counter-clockwise direction.

Note that the eigenloci are the loci of eigenvalues of $L(s)$. The advantage of the generalized Nyquist stability criterion is that instead of solving the Nyquist eigenloci of $\operatorname{det}(I+L(s))$, only plotting the eigenloci of $L(s)$ and counting the number of the encirclements of the $(-1+j 0)$ can directly obtain the complex system stability condition. The full mathematical proof of the generalized Nyquist criterion is given in [31].

To simplify, the sign " $\Delta$ " in the following paper is omitted. Considering the neutral time-delayed MG system (29), rewriting it in $s$-domain for a MIMO model yields

$s X_{M G}(s)-X_{M G_{0}}=A_{M G_{1}} X_{M G}(s)+e^{-t_{d} s} A_{M G_{2}} X_{M G}(s)+s e^{-t_{d} s} A_{M G_{3}} X_{M G}(s)$ where $X_{M G_{0}}$ is the initial value of $X_{M G}$ and rewriting (31) to obtain,

$$
X_{M G}(s)=\frac{1}{s}\left(I_{n}-\frac{1}{s}\left(A_{M G_{1}}+e^{-t_{d} s} A_{M G_{2}}+s e^{-t_{d} s} A_{M G_{3}}\right)\right)^{-1} X_{M G_{0}}
$$

Hence, the neutral time-delayed MG system can be modeled as shown in Fig. 3, with $r=X_{M G_{0}} ; y=X_{M G}(s) ; G(s)=I_{n} / s$ 


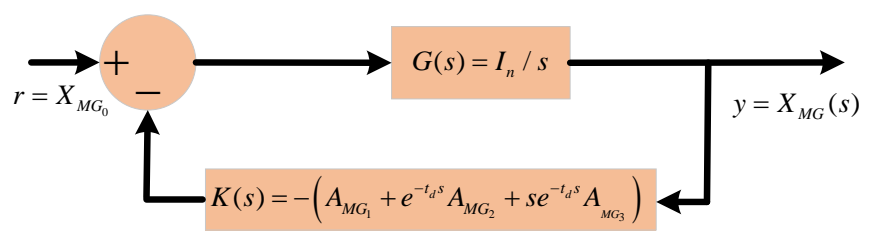

Fig. 3 MIMO time-delayed MG feedback system.

and $K(s)=-\left(A_{M G_{1}}+e^{-t_{d} s} A_{M G_{2}}+s e^{-t_{d} s} A_{M G_{3}}\right)$. Note that there are no unstable poles for $G(s)$ and $K(s)$, the neutral timedelayed MG system will be stable if and only if the eigenloci of $L(s)$ does not encircle the point $(-1+j 0)$.

Remark 5: For the MG small-signal model (29), the WAP length $\sigma$ has an upper boundary which is determined by stability condition. To guarantee the differential equation $f(x)=$ $\dot{X}_{M G}(t)-A_{M G_{3}} \dot{X}_{M G}\left(t-t_{d}\right)=0$ is asymptotically stable, the following equation needs to be met [32],

$$
\left\|A_{M G_{3}}\right\|<1
$$

By solving the equation (33), the maximum $\sigma$ can be obtained, which is a sufficient condition for system stability.

\section{B. Time-Domain Analytical Method for Time-Varying Delay Analysis}

When the time delay is time-varying, the time-delayed smallsignal model of (29) becomes,

$$
\dot{X}_{M G}(t)=A_{M G_{1}} X_{M G}(t)+A_{M G_{2}} X_{M G}\left(t-t_{d}(t)\right)+A_{M G_{3}} \dot{X}_{M G}\left(t-t_{d}(t)\right)
$$

To determine the TDM $\tau_{\max }$ and maximum time delay change rate (MTDCR) $\tau_{c}$, a time-domain method is proposed in this paper and the following theorem is introduced.

Theorem 2: For given scalars $\tau_{\max }>0$ and $\tau_{c}>0$, the timedelayed MG system (34) is asymptotically stable if there exist matrices $P>0, Q>0, X>0, Z>0, R>0$ and a matrix $Y$ satisfying the following LMIs [33],

$$
\begin{gathered}
{\left[\begin{array}{ccc}
\sum_{1,1} & \sum_{1,2} & \sum_{1,3} \\
* & \sum_{2,2} & \sum_{2,3} \\
* & * & \sum_{3,3}
\end{array}\right]<0} \\
{\left[\begin{array}{ccc}
X & Y \\
Y^{*} & \left(1-\tau_{c}\right) Z
\end{array}\right] \geq 0}
\end{gathered}
$$

where

$$
\begin{aligned}
& \sum_{1,1}=P A_{M G 1}+A_{M G 1}^{T} P+\tau_{\max } X+Y+Y^{T}+ \\
& A_{M G 1}^{T} Z A_{M G 1}+R+A_{M G 1}^{T} Q A_{M G 1} ; \\
& \sum_{1,2}=-Y+P A_{M G 2}+\tau_{\max } A_{M G 1}^{T} Z A_{M G 2}+A_{M G 1}^{T} Q A_{M G 2} ; \\
& \sum_{1,3}=P C+\tau_{\max } A_{M G 1}^{T} Z A_{M G 3}+A_{M G 1}^{T} Q A_{M G 3} ; \\
& \sum_{2,2}=-\left(1-\tau_{c}\right) R+\tau_{\max } A_{M G 2}^{T} Z A_{M G 2}+A_{M G 2}^{T} Q A_{M G 2} ; \\
& \sum_{2,3}=\tau_{\max } A_{M G 2}^{T} Z A_{M G 3}+A_{M G 2}^{T} Q A_{M G 3} ; \\
& \sum_{3,3}=-\left(1-\tau_{c}\right) Q+\tau_{\max } A_{M G 3}^{T} Z A_{M G 3}+A_{M G 3}^{T} Q A_{M G 3} .
\end{aligned}
$$

The detailed proof of Theorem 2 is shown in Appendix. By solving the LMI (35) and (36), a sufficient stability criterion can be obtained with a set of stability margin $\left\langle\tau_{\max }, \tau_{c}, \sigma\right\rangle$. The stability region can be used to guide the selection of WAP length $\sigma$. Note that for time-varying delay, the WAP length $\sigma$ is still required to meet the equation (33) to ensure the MG system stability.

\section{Improved WAP Length Tuning with a Higher Resolution}

To guarantee the system asymptotically stable, for control law (25), the WAP length $\sigma$ is constrained by (33) and the tuning range $\left[\begin{array}{ll}0 & \sigma_{\max }\end{array}\right]$ is relatively narrow. Taking the $\mathrm{MG}$ system in Fig. 4 as an example, the calculated $\sigma_{\max }$ is only 0.017. To relax the parameter sensitivity and provide a more accurate parameter tuning with a higher resolution, some nonlinear predictor coefficient functions for improving the tuning range are discussed and compared in this subsection.

Firstly, defining the linear function $\delta(\sigma)=\sigma$ for (25), then rewriting (25) as follow,

$\hat{x}\left(t-t_{d}+\delta(\sigma)\right)=x\left(t-t_{d}\right)+\delta(\sigma) \dot{x}\left(t-t_{d}\right) ; 0<\delta(\sigma)<t_{d}$

Then, three new non-linear $\delta_{i}(\sigma)$ are proposed to replace $\delta(\sigma)$ and the new control law becomes,

$\hat{x}\left(t-t_{d}+\delta_{i}(\sigma)\right)=x\left(t-t_{d}\right)+\delta_{i}(\sigma) \dot{x}\left(t-t_{d}\right) ; 0<\delta_{i}(\sigma)<t_{d}$

where

$\delta_{1}(\sigma)=0.02\left(e^{\sigma}-1\right)\left(1-[\tanh (\sigma)]^{2}\right)+0.02 * \tanh (\sigma)$;

$\delta_{2}(\sigma)=0.02 \tanh (\sigma)$

$\delta_{3}(\sigma)=0.02\left(1-\frac{\sigma}{e^{\sigma-1}}\right)$.

Instead of directly tuning the predictor parameter $\delta(\sigma)$, the proposed non-linear predictor coefficient functions adjust $\sigma$ to control $\delta(\sigma)$. Compared with a narrow interval of $\delta(\sigma) / \delta_{i}(\sigma)$, the tuning interval of $\sigma$ is wider when $\delta_{i}(\sigma)$ is implemented. Taking the MG system in Fig. 4 as an example, the comparison of the parameter tuning intervals for four predictor coefficient functions is shown in Table II.

From Table II, it can be noted that the tuning range of $\delta_{i}(\sigma)$ keep unchanged, but the interval of $\sigma$ will change to [0, 0.43], $[0,1.26]$ and $[0,3.07]$, which significantly improves the resolution of the predictor coefficient $\delta(\sigma)$. The gain for nonlinear predictor coefficient functions is designed as 0.02 in this case, which is $12 \%$ higher than the $\sigma_{\max }$. Fig. 5 shows the performance of four predictor coefficient functions. From the result, some general design principles are summarized for the predictor coefficient functions $\delta_{i}(\sigma)$.

\section{TABLE II COMPARISON OF THE PARAMETER TUNING INTERVALS FOR FOUR PREDICTOR COEFFICIENT FUNCTIONS}

\begin{tabular}{c|c|c}
\hline $\begin{array}{c}\text { Predictor coefficient } \\
\text { function }\end{array}$ & $\begin{array}{c}\text { Parameter interval } \\
\text { of } \delta(\sigma) / \delta_{i}(\sigma)\end{array}$ & $\begin{array}{c}\text { Tuning interval } \\
\text { of } \sigma\end{array}$ \\
\hline $\boldsymbol{\delta}(\boldsymbol{\sigma})$ & {$[0,0.017]$} & {$[0,0.017]$} \\
\hline $\boldsymbol{\delta}_{\mathbf{1}}(\boldsymbol{\sigma})$ & {$[0,0.017]$} & {$[0,0.43]$} \\
\hline $\boldsymbol{\delta}_{\mathbf{2}}(\boldsymbol{\sigma})$ & {$[0,0.017]$} & {$[0,1.26]$} \\
\hline $\boldsymbol{\delta}_{\mathbf{3}}(\boldsymbol{\sigma})$ & {$[0,0.017]$} & {$[0,3.07]$} \\
\hline
\end{tabular}

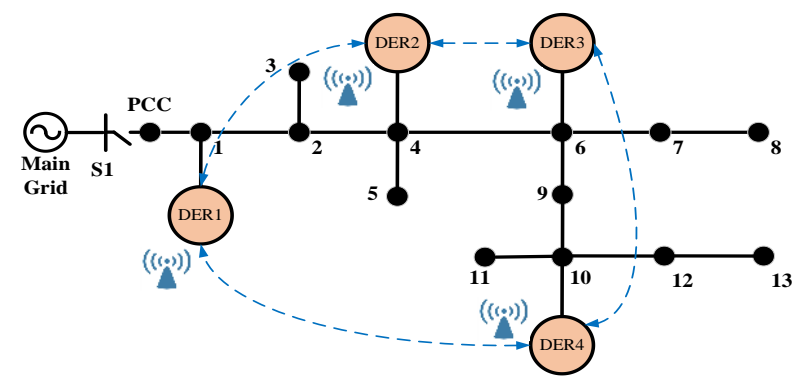

Fig. 4. Electrical structure and communication topology of an exemplarily 13 bus MG system 


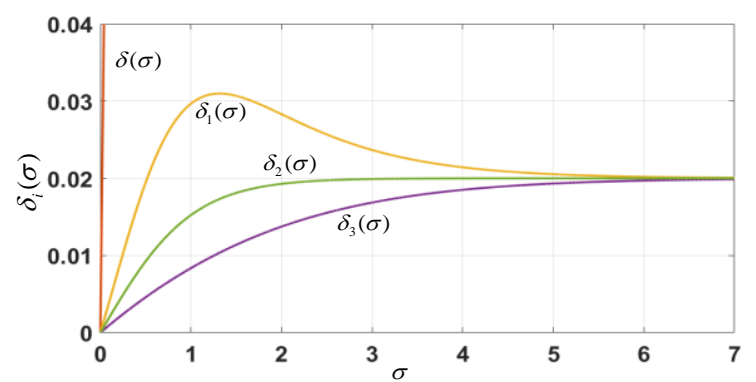

Fig. 5 The performance of four WAP coefficient functions

1) Compared with $\delta(\sigma)$ and $\delta_{1}(\sigma), \delta_{2}(\sigma)$ and $\delta_{3}(\sigma)$ would be better options if higher resolution is required. Especially, $\delta_{3}(\sigma)$ has a higher resolution than $\delta_{2}(\sigma)$ but $\delta_{2}(\sigma)$ has a faster compensation effect.

2) $\delta(\sigma)$ and $\delta_{1}(\sigma)$ could rapidly increase the predictive effect. However, the stability will be impacted in the case of exceeding $\sigma$.

3) For $\delta_{i}(\sigma), i=1,2,3$, as $\sigma$ increases to a certain value, the performance is commensurate.

\section{TEST RESUlts}

In this paper, OPAL-RT is used to perform a real-time test of the proposed control methods. An islanded 13-bus MG test system as shown in Fig. 4 is built in RTLAB and the real-time test setup is shown in Fig. 6. The OPAL-RT test results are exported by a LeCroy oscilloscope. Three equivalent loads are connected to bus 5,8 and 11 , respectively. The system parameters are presented in TABLE III. Noted that the converter IGBT-switch model is adopted in the time-domain validation. Besides, the initial equilibrium point of the smallsignal model is shown in Table IV.

As shown in Fig. 4, the AC MG contains 4 DERs and each DER connects to the bus by resistive-inductive tie-lines. The isolated switch $S_{1}$ is open. The communication topology is strongly connected as shown in the blue line of Fig. 4. Such an MG architecture is mainly applied in the isolated island MG system such as Renewable Energy Integration DemonstratorSingapore (REIDS) testbed in Semakau island, Singapore [34].

\section{A. Model Validation: Barely Stable and Unstable Case}

With the above sample MG system and system parameters, the system matrices $A_{M G_{1}}, A_{M G_{2}}, A_{M G_{3}}$ can be obtained. Note $A_{M G_{3}}$ is a $\sigma$-related matrix and one can obtain the maximum $\sigma=0.017$ according to (33). In this validation case, the WAP length $\sigma$ is set as 0.012 . The effectiveness of the proposed Nyquist-based analytical method is verified by timedomain simulation in MATLAB/SimPowerSystems. Considering the barely stable and unstable condition, Fig. 7 shows the Nyquist plots with the fixed time delay $t_{d}=54 \mathrm{~ms}$ and $t_{d}=55 \mathrm{~ms}$. The left part is an overview of the Nyquist contours, and the right part is the zoom-in of the point $(-1+j 0)$.

From Fig. 7, it shows the eigenloci of $L(s)$ will encircle the point $(-1+\mathrm{j} 0)$ when the $t_{d}$ increases from $54 \mathrm{~ms}$ to $55 \mathrm{~ms}$. According to Theorem 1, due to no unstable poles for $G(s)$ and $K(s)$, the encirclement will cause system instability. The timedomain experiment results in Fig. 8 verify the conclusion, which also proves the accuracy of the developed time- delayed small-signal model and the effectiveness of the proposed graphical analytical method.

TABle III Parameters OF THE MG System

\begin{tabular}{|c|c|c|}
\hline Variable & Value & Unit \\
\hline Inverter LCL filter $\boldsymbol{L}_{\boldsymbol{f}}, \boldsymbol{C}_{\boldsymbol{f}}, \boldsymbol{L}_{\boldsymbol{o}}$ & {$\left[\begin{array}{lll}1.8 & 25 & 1.8\end{array}\right]$} & $m H, \mu F, m H$ \\
\hline Inverter RL output connecter $\boldsymbol{Z}_{l}$ & $0.2+j 1$ & $\Omega, m H$ \\
\hline $\begin{array}{l}\text { Equivalent Line Impendence } \\
\boldsymbol{Z}_{l_{12},}, \boldsymbol{Z}_{l_{23},} \boldsymbol{Z}_{\boldsymbol{l Z 4}}\end{array}$ & $\begin{array}{c}{[0.1+\mathrm{j} 0.1} \\
0.2+\mathrm{j} 0.10 .1+\mathrm{j} 0.2]\end{array}$ & $\Omega, m H$ \\
\hline Power filter cutoff-frequency $\omega_{c}$ & 10 & $\mathrm{rad} / \mathrm{s}$ \\
\hline Frequency-droop coefficient $\boldsymbol{k}_{p 1-2}$ & $1 \mathrm{e}-4$ & $\mathrm{rad} / \mathrm{s} / \mathrm{W}$ \\
\hline Frequency-droop coefficient $\boldsymbol{k}_{p 3-4}$ & $5 e-5$ & $\mathrm{rad} / \mathrm{s} / \mathrm{W}$ \\
\hline Voltage-droop coefficient $\boldsymbol{k}_{q 1-2}$ & $2 \mathrm{e}-4$ & V/Var \\
\hline Voltage-droop coefficient $\boldsymbol{k}_{\boldsymbol{q} 3-4}$ & $1 \mathrm{e}-4$ & $V / V a r$ \\
\hline Secondary controller $\boldsymbol{c}_{\omega}, \boldsymbol{c}_{p}, \boldsymbol{c}_{v}, \boldsymbol{c}_{\boldsymbol{q}}$ & {$\left[\begin{array}{llll}10 & 1 & 10 & 0.1\end{array}\right]$} & \\
\hline $\begin{array}{c}\text { Voltage loop proportional gain } \boldsymbol{k}_{v p} \\
\text { integral gain } \boldsymbol{k}_{v i}\end{array}$ & [4 40] & \\
\hline $\begin{array}{l}\text { Current loop proportional gain } \boldsymbol{k}_{i p}, \\
\text { integral gain } \boldsymbol{k}_{\boldsymbol{i} i}\end{array}$ & {$\left[\begin{array}{lll}0.05 & 10\end{array}\right]$} & \\
\hline Load-1 & $5+j 5$ & $k W, k V a r$ \\
\hline Load-2 & $10+j 5$ & $k W, k \operatorname{Var}$ \\
\hline Load-3 & $15+j 5$ & $k W, k V a r$ \\
\hline Load-4 & $13+j 12$ & $k W, k V a r$ \\
\hline Nominal frequency\& voltage & {$\left[\begin{array}{lll}314.15 & 311.1\end{array}\right]$} & $\mathrm{rad} / \mathrm{s}, \mathrm{V}$ \\
\hline DC voltage & 800 & $V$ \\
\hline Sampling time & $1 \mathrm{e}-5$ & $s$ \\
\hline Switching frequency & 7 & $\mathrm{kHz}$ \\
\hline
\end{tabular}

TABle IV MG System InITIAL EquiLIBRIUM PoINT

\begin{tabular}{|c|c|c|c|}
\hline Par. & Value & Par. & Value \\
\hline $\boldsymbol{V}_{\boldsymbol{r d}}(V)$ & $\begin{array}{c}{\left[\begin{array}{lll}311.1 & 311.1 \\
311.1 & 311.1]\end{array}\right.}\end{array}$ & $\delta_{0}(r a d)$ & $\begin{array}{c}{[0-0.0220 .0089} \\
0.0043]\end{array}$ \\
\hline$I_{o d}(A)$ & $\begin{array}{c}{\left[\begin{array}{ll}10.89 & 10.87 \\
21.86 & 21.84\end{array}\right]} \\
\end{array}$ & $I_{o q}(A)$ & $\begin{array}{c}{[-4.499-8.414-8.572} \\
-11.99]\end{array}$ \\
\hline$I_{\text {line12d }}(A)$ & [10.89] & $I_{\text {line12q }}(A)$ & [-4.499] \\
\hline$I_{\text {line } 23 d}(A)$ & [10.97] & $I_{\text {line } 23 q}(A)$ & {$[-1.947]$} \\
\hline$I_{\text {line } 34 d}(A)$ & [11.01] & $I_{\text {line } 34 q}(A)$ & [0.6299] \\
\hline$I_{\text {load1d }}(A)$ & [10.80] & $I_{\text {load1q }}(A)$ & {$[-10.97]$} \\
\hline$I_{\text {load } 2 d}(A)$ & [21.82] & $I_{\text {load } 2 q}(A)$ & {$[-11.15]$} \\
\hline$I_{\text {load } 3 d}(A)$ & [32.85] & $I_{\text {load } 3 q}(A)$ & {$[-11.36]$} \\
\hline$\omega_{0}(\mathrm{rad} / \mathrm{s})$ & \multicolumn{3}{|c|}{ [314.159] } \\
\hline
\end{tabular}
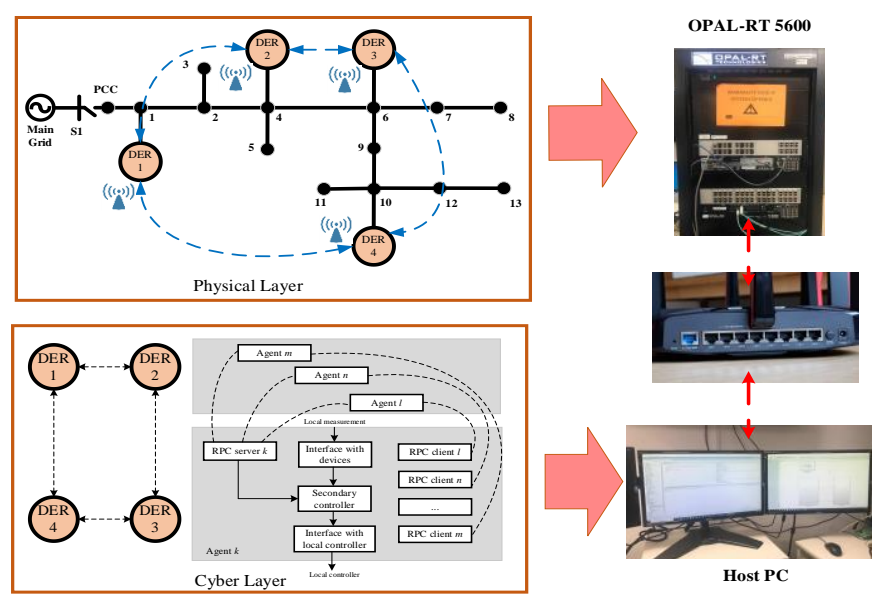

Fig. 6. Real-time test simulation based on OPAL-RT OP5600. 


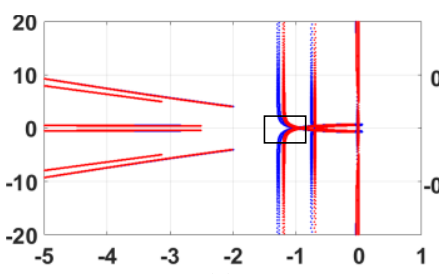

(a)

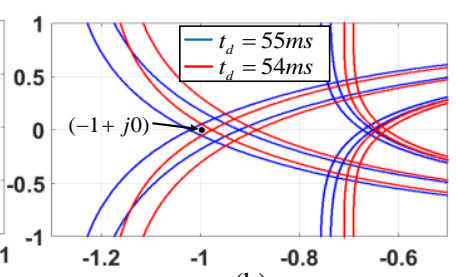

(b)
Fig. 7 Nyquist plots with $t_{d}=54 \mathrm{~ms}$ and $t_{d}=55 \mathrm{~ms}$. (a) Overview of the Nyquist contours. (b) Zoom-in of the point $(-1+\mathrm{j} 0)$.

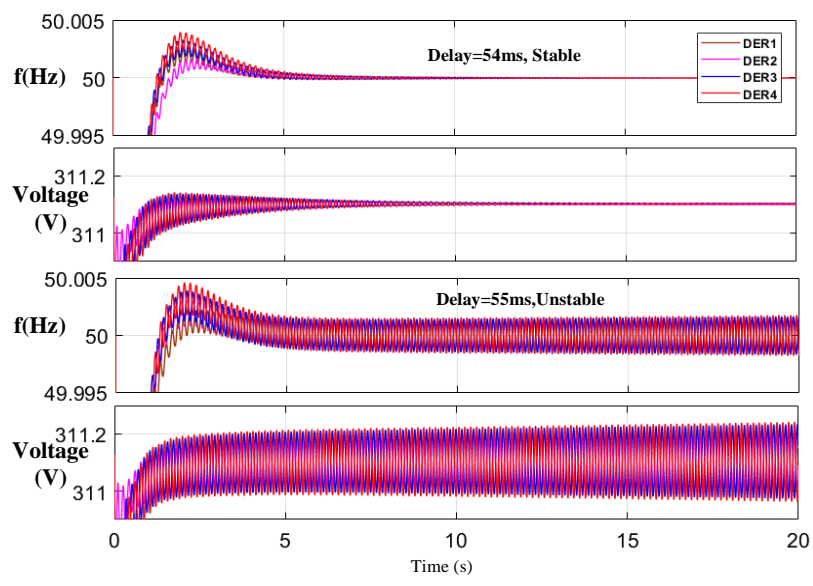

Fig. $8 \mathrm{f} / \mathrm{V}$ time-domain performance with $t_{d}=54 \mathrm{~ms}$ and $t_{d}=55 \mathrm{~ms}$

\section{B. Stability Validation under Fixed Delay}

In this section, the routing delay and serial delay are assumed as a certain delay. Based on the proposed graphical analytical method, the impact of different WAP length $\sigma$ is analyzed. Fig. 9 shows the relationship between the WAP length $\sigma$ and the maximum delay $\tau^{*}$.

From the result, it is noticeable that the maximum stability margin $\tau^{*}$ increases within the feasible interval of the WAP length $\sigma$, which reveals that the proposed control method can improve the TDM and thus improves the system robustness to communication delay. The stability region shown in Fig. 9 provides a feasible region to guide the selection of the system parameter $\sigma$. However, note that the WAP length $\sigma$ has an upper boundary which is determined by (33). The excessive $\sigma$ will cause the system instability for the arbitrary delay. Furthermore, when $\delta(\sigma)=\sigma$ for (37), the tuning range of $\sigma$ for exemplary MG is [0, 0.017]. If $\delta_{1}(\sigma), \delta_{2}(\sigma)$ and $\delta_{3}(\sigma)$ are selected, the tuning range of $\delta_{i}(\sigma)$ keep unchanged, but the interval of $\sigma$ will change to [0, 0.43], [0, 1.26] and [0,3.07], which significantly improves the resolution of WAP length $\sigma$.

To validate the above stability analysis, a set of time-domain experiments in the OPAL-RT experiment platform are carried out. In this case, the internal controllers and the LCL output filters were included in OPAL-RT tests. Three points including A, B, C in Fig. 9 are selected, and the experiment results of the voltage and frequency output are depicted in Fig. 10. Note that when $\sigma=0$, the control law (20) and (21) is equivalent to (27) and (28). It can be seen that system in point $\mathbf{A}$ is unstable while it is stable in point $\mathbf{B}$, which validates the conclusion that WAP controller can enhance the TDM for MG system. Note that MG system in point $\mathbf{C}$ is unstable even the delay is only $44 \mathrm{~ms}$, which proves the existence of upper boundary of the WAP length $\sigma$.

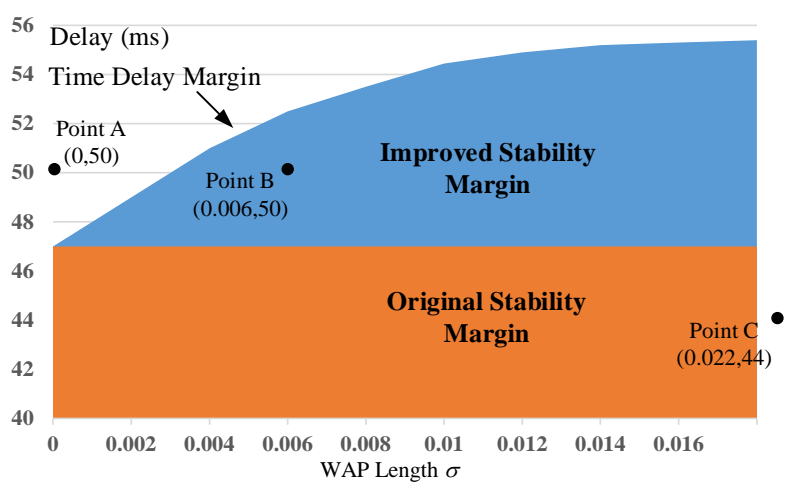

Fig. 9 The TDM comparison between Eq. $6 \& 7$ and Eq. $23 \& 24$
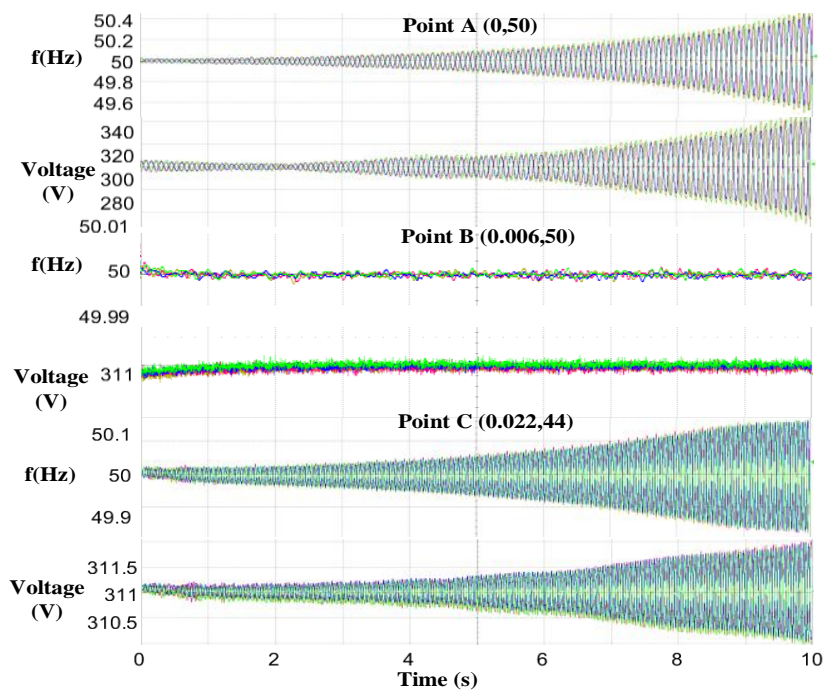

Fig. $10 \mathrm{f} \mathrm{\& V}$ time-domain performance with point A, B and C

\section{Stability Validation under the Time-Varying Delay}

Unlike the fixed time delay, the time-varying delay is more common in the practical MG communication with the uncertain routing delay and serial delay. Two indexes including TDM and MTDCR need to be considered. In this case, a fixed delay $\tau_{f}$ is to emulate the certain packet delay and propagation delay and a sinusoidal time-varying delay $\tau_{\max } \sin \left(\omega_{c} t\right)$ is used to emulate the uncertain routing delay and serial delay. The MTDCR can be obtained by $\tau_{c}=\tau_{\max } * \omega_{c}$. In this subsection, the relationship among TDM, TDMCR and WAP length $\sigma$ are studied. By keeping $\tau_{f}=20 \mathrm{~ms}$ unchanged, the system characteristics determined by $\tau_{\max }, \tau_{c}$, and WAP length $\sigma$ are analyzed. The above time-domain analytical method provides a set of stability boundaries $\left\langle\sigma, \tau_{\max }, \tau_{c}\right\rangle$.

To show the relationship among $\tau_{\max }, \tau_{c}$ and WAP length $\sigma$, three sets of time-domain experiments are carried out to compare stability boundaries with the results shown in Fig. 11. For time-varying delay with $\sigma=0$, note that the MG system has a higher TDM (85ms) compared with the fixed delay $(47 \mathrm{~ms})$. The reason is the time-varying delay changes from zero to the TDM and hence the system can tolerate a higher TDM for a wide time spectrum. However, the system is hard to keep stable if the time delay is always fixed as TDM. Hence, compared with the delay randomly changing from zero to TDM, fixed delay with the same TDM can be regarded as the worstcase scenario. Besides, it can be seen, the TDMCR gradually 


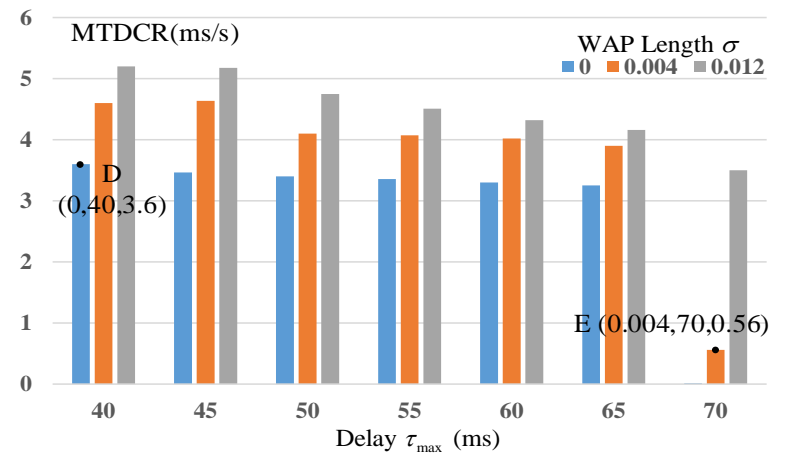

Fig. 11 The stability boundary comparison under time-varying delays with $\tau_{f}=20 \mathrm{~ms}$

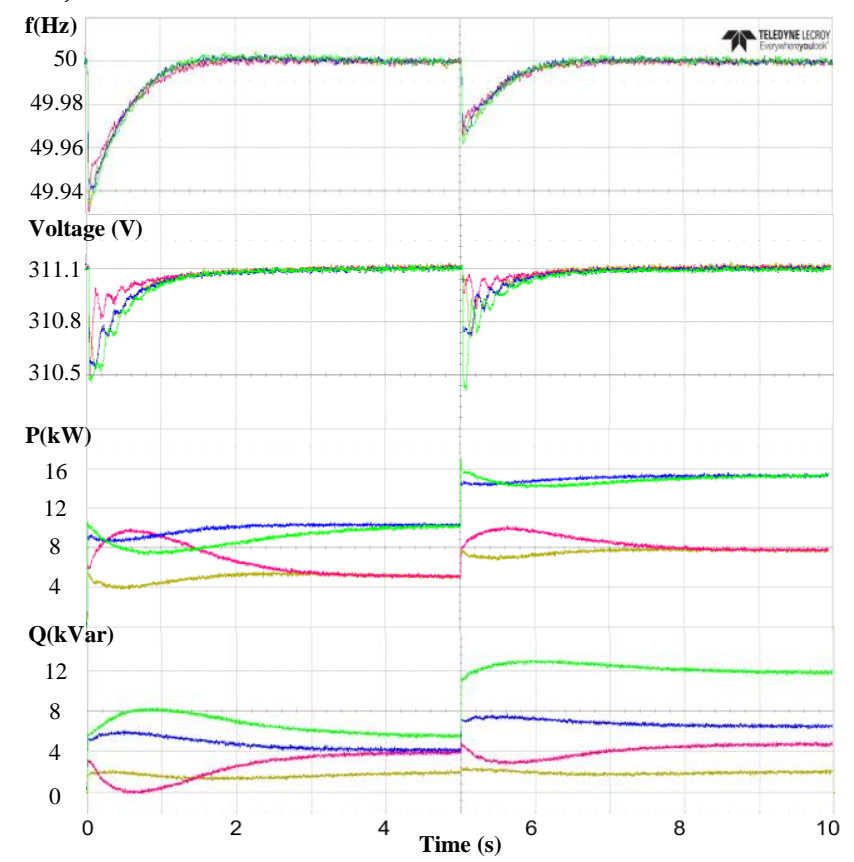

Fig. 12 f/V/P/Q time-domain performance under delay $|0.04 \sin (90 t)|$ with $\sigma=0$ (D point)

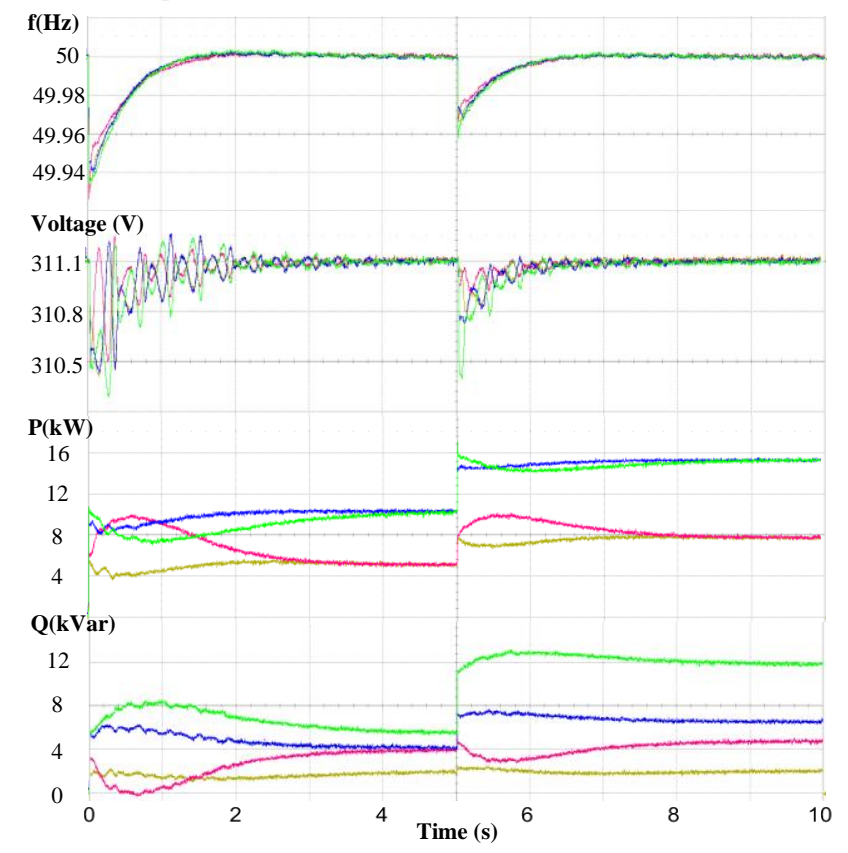

Fig. $13 \mathrm{f} / \mathrm{V} / \mathrm{P} / \mathrm{Q}$ time-domain performance under delay $|0.07 \sin (8 t)|$ with $\sigma=$ 0.004 (E point)

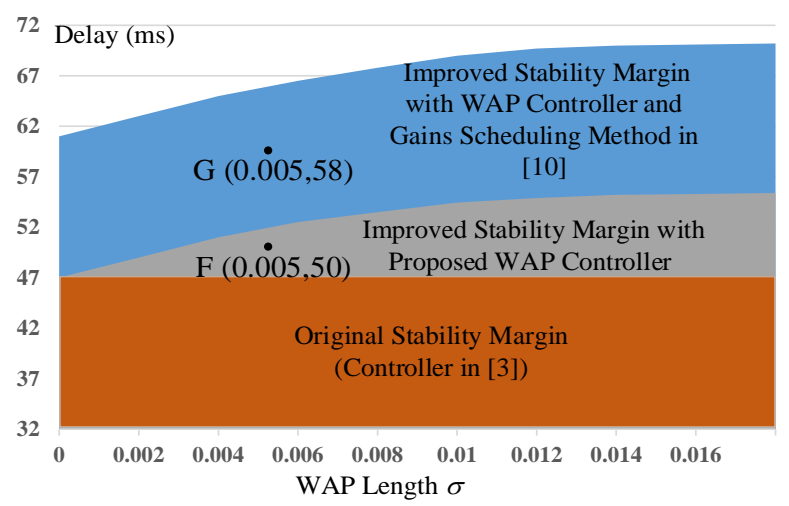

Fig. 14 The TDM comparison among controllers in [3], [10] and this paper

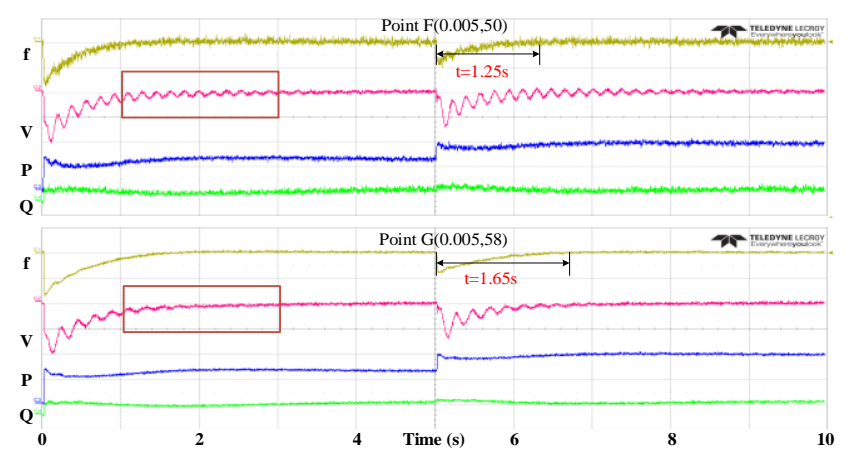

Fig. $15 \mathrm{The} / \mathrm{V} / \mathrm{P} / \mathrm{Q}$ time-domain performance with $\sigma=0.005$ (F and $\mathrm{G}$ point)

decreases with the increase of delay but the increased WAP length $\sigma$ will boost the TDM and TDMCR, which verifies the effectiveness of the proposed WAP controller again.

Another two points $\mathbf{D}(0,40,3.6)$ and $\mathbf{E}(0.004,70,0.56)$ in the critical stable boundary are selected to validate the analytical result and the performance with different time delay and change rates are compared in Fig. 12 and Fig. 13, respectively. A load step change in $\mathrm{t}=5 \mathrm{~s}$ is considered. The results show the frequency and voltage can be restored and the active power is also accurately shared (1:1:2:2). Besides, Fig. 12 and Fig. 13 reveal that the system oscillation depends on the delay change rate and the lower MTDCR (E Point) will cause a more significant pulse effect.

\section{Comparison \& Cooperation with Gain-Scheduling Method}

The gain-scheduling method is a practical and simple method to significantly increase the TDM, which has been widely investigated and validated such as in [10] and [26].

Based on the conclusion in [10], the frequency/voltage integral gains $c_{f}$ and $c_{v}$ decreases from 10 to 8 , respectively. The other parameters keep the same and the new stability margin is shown in Fig. 14. Another two points $\mathbf{F}(0.005,40.5)$ and $\mathbf{G}(0.005,43)$ in the different stability fields are selected to validate the analytical result. The performance with different gains is shown in Fig. 15. The same load -4 step change at $\mathrm{t}=5 \mathrm{~s}$ is considered. From Figs. 14 and 15, some conclusions can be concluded: 1) The gain-scheduling method have a better performance due to a bigger improved stable area (both blue area and gray area). 2) Although gain-scheduling method has a better performance against time delay, it sacrifices the system convergence speed. 3) The TDM can be further improved by the cooperation between the proposed WAP controller and the gain-scheduling method. 

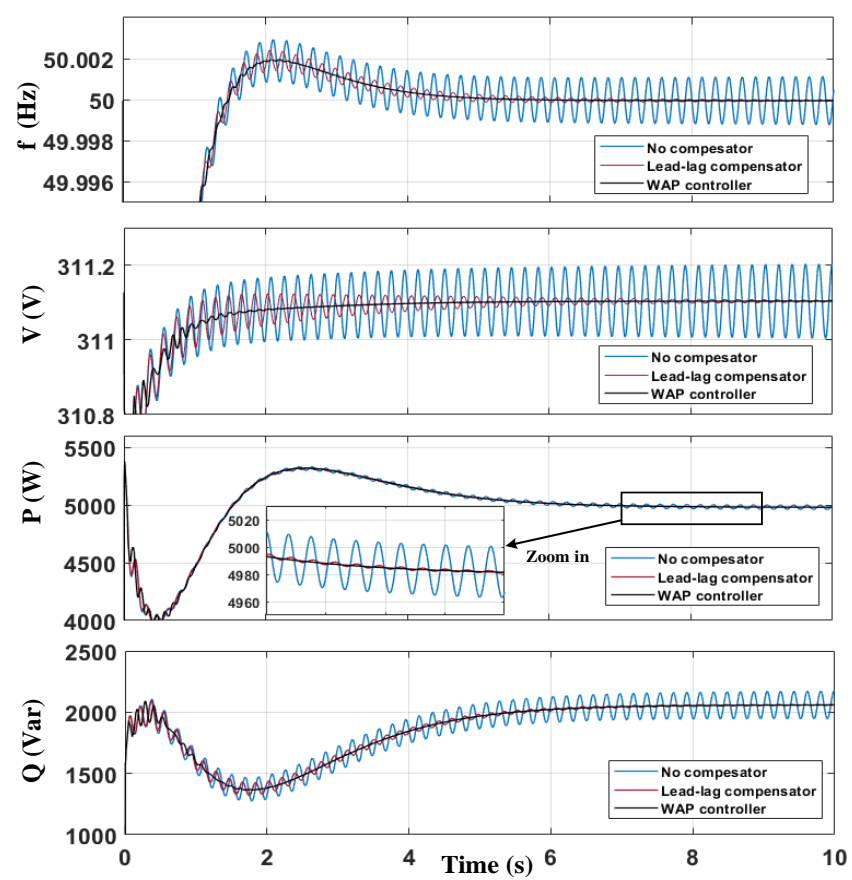

Fig. 16 The f/V/P/Q time-domain performance considering no predictor/compensator, lead-lag compensator (33) and proposed WAP controller.

Fig. 15 validates the above conclusions by the selected point $\mathrm{F}$ and $\mathrm{G}$. It can be seen, gain-scheduling further improve the TDM from the only gray area to both gray and bule area. However, point $\mathbf{G}$ has a slower convergence speed (1.65s) than it of point $\mathbf{F}$ (1.25s) due to the decrease of frequency/voltage integral gains. Moreover, the system has a higher oscillation in $\mathbf{F}$ point than it in $\mathbf{G}$ point (shown in red frame) because $\mathbf{F}$ point is closer to stability boundary than G point. The OPAL-RT experiment results validate the effectiveness of the proposed WAP method. Besides, the cooperation with gain-scheduling method is simple and flexible in a practical MG system.

\section{E. Comparison Study with Lead-Lag Compensator}

In this subsection, the performance of the proposed WAP controller is further validated through a comparison study using MATLAB/SimPowerSystems toolbox. The proposed control method is compared with the lead-lag compensator proposed in [28]. The lead-lag compensator is based on the frequency domain analysis, which can provide phase lead to compensate the phase lag caused by time delay and thus improve the system performance against time delay. Typically, the compensator is designed as a transfer function, which adds to all communication signals or data from neighbors before utilized by controllers.

In this case, based on the design principle of compensator in [28], a second-order lead-lag block is chosen in (39) to provide more phase lead compared with a first order one, the lead-lag compensator is designed as follow,

$$
H_{c}(s)=\frac{\left(T_{c 1} s+1\right)^{2}}{\left(T_{c 2} s+1\right)^{2}}
$$

where $T_{c 1}$ and $T_{c 2}$ are the time constants of the lead-lag block. The time constants are selected as $T_{c 1}=0.002 \mathrm{~s}$ and $T_{c 2}=$ $0.0003 \mathrm{~s}$ based on the maximum phase lead $\varphi_{m}$ and its corresponding frequency $\omega_{m}$. The delay is set as $48 \mathrm{~ms}$ and the
WAP length $\sigma$ is chosen as 0.006 for the proposed WAP controller. Taking DER 1 as an example, the performance comparison considering no predictor/compensator [3], lead-lag compensator [28] and proposed WAP controller are shown in Fig. 16. It can be seen, under a fixed delay of $48 \mathrm{~ms}$, the system is near critical stable if no compensator/predictor is implemented. However, both the lead-lag compensator and proposed WAP controller can significantly improve the system performance and the oscillations of system state are much damped. Compared with the lead-lag compensator, the proposed WAP controller has a better performance with fewer oscillations. Moreover, the lead-lag compensator only deals with fixed time-delay issues because it is designed based on the frequency-domain analysis, the proposed WAP controller can be further applied to the scenario under time-varying delay. The effectiveness is validated in the above time-domain analysis and experiments.

\section{CONCLUSION}

This paper works on the MG system with communication time delay. A novel distributed control method is proposed for the counteraction of the communication delay effect. Based on a developed small-signal model, an innovative graphic analytical method based on the generalized Nyquist theorem and a time-domain Lyapunov method are proposed for the stability study. The OPAL-RT simulation tests validate the effectiveness of the proposed controllers in both fixed time delay and timevarying delay scenarios. In addition, the performance of the proposed controller is compared with lead-lag compensator.

\section{APPENDIX}

\section{Stability Proof of Theorem 2}

To prove Theorem 2, a Lyapunov function is introduced for MG system (34) as follows,

where

$$
V=V_{1}+V_{2}+V_{3}+V_{4}
$$

where

$$
\begin{gathered}
V_{1}=X_{M G}^{T}(t) P X_{M G}(t) \\
V_{2}=\int_{-t_{d}(t)}^{0} \int_{t+\beta}^{t} \dot{X}_{M G}^{T}(\alpha) Z \dot{X}_{M G}(\alpha) d \alpha d \beta \\
V_{3}=\int_{t-t_{d}(t)}^{t} X_{M G}^{T}(\alpha) R X_{M G}(\alpha) d \alpha \\
V_{4}=\int_{t-t_{d}(t)}^{t} \dot{X}_{M G}^{T}(\alpha) Q \dot{X}_{M G}(\alpha) d \alpha
\end{gathered}
$$

Taking the time derivative of $V$, we have

$$
\dot{V}_{1}=\dot{X}_{M G}^{T}(t) P X_{M G}(t)+X_{M G}^{T}(t) P \dot{X}_{M G}(t)
$$

Rewriting the MG system model (34) to obtain

$$
\begin{aligned}
& \dot{X}_{M G}(t)=\left(A_{M G_{1}}+A_{M G_{2}}\right) X_{M G}(t) \\
& \quad-A_{M G_{2}} \int_{t-t_{d}(t)}^{t} \dot{X}_{M G}(\alpha) d \alpha+A_{M G_{3}} \dot{X}_{M G}\left(t-t_{d}(t)\right)
\end{aligned}
$$

Based on (46), we have

$$
\begin{aligned}
\dot{V}_{1} & =2 X_{M G}^{T}(t) P\left(A_{M G_{1}}+A_{M G_{2}}\right) X_{M G}(t) \\
& -2 X_{M G}^{T}(t) P A_{M G_{2}} \int_{t-t_{d}(t)}^{t} \dot{X}_{M G}(\alpha) d \alpha \\
& +2 X_{M G}^{T}(t) P A_{M G_{3}} \dot{X}_{M G}\left(t-t_{d}(t)\right)
\end{aligned}
$$

Applying the inequality (17) in [33], we have 


$$
\begin{aligned}
\dot{V}_{1} \leq & 2 X_{M G}^{T}(t) P\left(A_{M G_{1}}+A_{M G_{2}}\right) X_{M G}(t) \\
& +\tau_{\max } X_{M G}^{T}(t) X X_{M G}(t) \\
& +2 X_{M G}^{T}(t)\left(Y-P A_{M G_{2}}\right) \int_{t-t_{d}(t)}^{t} \dot{X}_{M G}(\alpha) d \alpha \\
& +\left(1-\tau_{c}\right) \int_{t-t_{d}(t)}^{t} \dot{X}_{M G}^{T}(\alpha) Z \dot{X}_{M G}(\alpha) d \alpha \\
& +2 X_{M G}^{T}(t) P A_{M G_{3}} \dot{X}_{M G}\left(t-t_{d}(t)\right) \\
= & X_{M G}^{T}(t)\left(A_{M G_{1}}^{T} P+P A_{M G_{1}}+Y+Y^{T}+\tau_{\max } X\right) X_{M G}(t) \\
& -2 X_{M G}^{T}(t)\left(Y-P A_{M G_{2}}\right) X_{M G}\left(t-t_{d}(t)\right) \\
& +\left(1-\tau_{c}\right) \int_{t-t_{d}(t)}^{t} \dot{X}_{M G}^{T}(\alpha) Z \dot{X}_{M G}(\alpha) d \alpha \\
& +2 X_{M G}^{T}(t) P A_{M G_{3}} \dot{X}_{M G}\left(t-t_{d}(t)\right)
\end{aligned}
$$

Similarly, we write the time derivative of $V_{2}, V_{3}$ and $V_{4}$,

$$
\begin{aligned}
\dot{V}_{2}= & t_{d}(t) \dot{X}_{M G}^{T}(t) Z \dot{X}_{M G}(t) \\
& -\left(1-t_{d}(t)\right) \int_{t-t_{d}(t)}^{t} \dot{X}_{M G}^{T}(\alpha) Z \dot{X}_{M G}(\alpha) d \alpha \\
\leq & \tau_{\max } \dot{X}_{M G}^{T}(t) Z \dot{X}_{M G}(t) \\
& -\left(1-\tau_{c}\right) \int_{t-t_{d}(t)}^{t} \dot{X}_{M G}^{T}(\alpha) Z \dot{X}_{M G}(\alpha) d \alpha \\
\leq & {\left[A_{M G_{1}} X_{M G}(t)+A_{M G_{2}} X_{M G}\left(t-t_{d}(t)\right)+A_{M G_{3}} \dot{X}_{M G}\left(t-t_{d}(t)\right)\right]^{T} \tau_{\max } } \\
& Z\left[A_{M G_{1}} X_{M G}(t)+A_{M G_{2}} X_{M G}\left(t-t_{d}(t)\right)+A_{M G_{3}} \dot{X}_{M G}\left(t-t_{d}(t)\right)\right] \\
& -\left(1-\tau_{c}\right) \int_{t-t_{d}(t)}^{t} \dot{X}_{M G}^{T}(\alpha) Z \dot{X}_{M G}(\alpha) d \alpha
\end{aligned}
$$$$
\dot{V}_{3}=X_{M G}^{T}(t) R X_{M G}(t)
$$$$
-\left(1-\dot{t}_{d}(t)\right) X_{M G}^{T}\left(t-t_{d}(t)\right) R X_{M G}\left(t-t_{d}(t)\right)
$$$$
\leq X_{M G}^{T}(t) R X_{M G}(t)
$$$$
-\left(1-\tau_{c}\right) X_{M G}^{T}\left(t-t_{d}(t)\right) R X_{M G}\left(t-t_{d}(t)\right)
$$$$
\dot{V}_{4}=\dot{X}_{M G}^{T}(t) Q \dot{X}_{M G}(t)
$$$$
-\left(1-\dot{t}_{d}(t)\right) \dot{X}_{M G}^{T}\left(t-t_{d}(t)\right) Q \dot{X}_{M G}\left(t-t_{d}(t)\right)
$$$$
\leq \dot{X}_{M G}^{T}(t) Q \dot{X}_{M G}(t)
$$$$
-\left(1-\tau_{c}\right) \dot{X}_{M G}^{T}\left(t-t_{d}(t)\right) Q \dot{X}_{M G}\left(t-t_{d}(t)\right)
$$$$
=\left[A_{M G_{1}} X_{M G}(t)+A_{M G_{2}} X_{M G}\left(t-t_{d}(t)\right)+A_{M G_{3}} \dot{X}_{M G}\left(t-t_{d}(t)\right)\right]^{T}
$$$$
Q\left[A_{M G_{1}} X_{M G}(t)+A_{M G_{2}} X_{M G}\left(t-t_{d}(t)\right)+A_{M G_{3}} \dot{X}_{M G}\left(t-t_{d}(t)\right)\right]
$$$$
-\left(1-\tau_{c}\right) \dot{X}_{M G}^{T}\left(t-t_{d}(t)\right) Q \dot{X}_{M G}\left(t-t_{d}(t)\right)
$$

From (48)-(51), we have

$$
\dot{V}=\dot{V}_{1}+\dot{V}_{2}+\dot{V}_{3}+\dot{V}_{4} \leq \Pi^{T} \sum(P, Q, X, Y, Z) \Pi
$$

where $\Pi=\left[X_{M G}^{T}(t) X_{M G}^{T}\left(t-t_{d}(t)\right) \dot{X}_{M G}^{T}\left(t-t_{d}(t)\right)\right]$. If the LMI conditions (35) and (36) hold, then $\dot{V}<0$ and the neutral time-delayed MG system is asymptotic stable, which completes the proof.

\section{REFERENCES}

[1] D. E. Olivares et al., "Trends in microgrid control," IEEE Trans. Smart Grid, vol. 5, no. 4, pp. 1905-1919, Jul. 2014.

[2] J. Rocabert, A. Luna, F. Blaabjerg, and P. Rodriguez, "Control of power converters in AC microgrids," IEEE Trans. Power Electron., vol. 27, no. 11, pp. 4734-4749, Nov. 2012.
[3] F. Guo, et al., "Distributed secondary voltage and frequency restoration control of droop-controlled inverter-based microgrids," IEEE Trans. Ind. Electron., vol. 62, no. 7, pp. 4355-4364, Jul. 2015.

[4] J. M. Guerrero et al., "Hierarchical control of droop-controlled AC and DC microgrids-A general approach toward standardization," IEEE Trans. Ind. Electron., vol. 58, no.1, pp. 158-172, Jan. 2011.

[5] Y. Wang, et al., "Cyber-physical design and implementation of distributed event-triggered secondary control in islanded microgrids," IEEE Trans. Ind. Appl., Aug. 2019.

[6] G. Lou, et al., "Distributed MPC based secondary voltage control scheme for autonomous droop-controlled microgrids, "IEEE Trans. Sustain. Energy, vol. 8, no. 2, pp. 792-804, Apr. 2017.

[7] J. Lai, H. Zhou, X. Lu, and Z. Liu, "Distributed power control for DERs based on networked multiagent systems with communication delays," Neurocomputing, vol. 179, pp. 135-143, Feb. 2016.

[8] V. P. Singh, N. Kishor, and P. Samuel, "Load frequency control with communication topology changes in smart grid," IEEE Trans. Ind. Inform., vol. 12, no. 5, pp. 1943-1952, Oct. 2016.

[9] Q. Zhou et al., "Distributed control and communication strategies in networked microgrids," IEEE Communications Surveys \& Tutorials, vol. 22, no. 4, pp. 2586-2633, Sep. 2020.

[10] S. Liu, X. Wang, and P. X. Liu, "Impact of communication delays on secondary frequency control in an islanded microgrid," IEEE Trans. Ind. Electron., vol. 62, no. 4, pp. 2021-2031, Nov. 2014.

[11] E. A. A. Coelho et al., "Small-signal analysis of the microgrid secondary control considering a communication time delay," IEEE Trans. Ind. Electron., vol. 63, no. 10, pp. 6257-6269, Oct. 2016.

[12] G. Lou et al., "Stability robustness for secondary voltage control in autonomous microgrids with consideration of communication delays," IEEE Trans. Power Syst., vol. 33, no. 4, pp. 4164-4178, Dec. 2017.

[13] H. Jia and X. Yu, "A simple method for power system stability analysis with multiple time delays," in Proc. IEEE Power and Energy Society General Meeting-Conversion and Delivery of Electrical Energy in the 21st Century, Pittsburgh, PA, USA, 2008.

[14] J. Lai, X. Lu, and X. Yu, "Stochastic distributed frequency and load sharing control for microgrids with communication delays," IEEE Syst. J., vol. 13, no. 4, pp. 4269-4280, Mar. 2019.

[15] H. R. Baghaee, M. Mirsalim, G. B. Gharehpetian, and H. A. Talebi, "A generalized descriptor-system robust $\mathrm{H} \infty$ control of autonomous microgrids to improve small and large signal stability considering communication delays and load nonlinearities," Int. J. Elect. Power Energy Syst., vol. 92, pp. 63-82, Nov. 2017.

[16] J. Lai, H. Zhou, X. Lu, X. Yu, and W. Hu, "Droop-based distributed cooperative control for microgrids with time-varying delays," IEEE Trans. Smart Grid, vol. 7, no. 4, pp. 1775-1789, Apr. 2016.

[17] F. Milano and M. Anghel, "Impact of time delays on power system stability," IEEE Trans. Circuits and Syst. I- Regul. Pap., vol. 59, no. 4, pp. 889-900, Oct. 2011.

[18] J. Sun, G. Liu, and J. Chen, "Delay-dependent stability and stabilization of neutral delay systems," Int. J. Robust Nonlinear Control, vol. 19, no. 12, pp. 1364-1375, Oct. 2008.

[19] M. Dong, et al., "stability analysis of a novel distributed secondary control considering communication delay in DC microgrids," IEEE Trans. Smart Grid, vol. 10, no. 6, pp. 6690-6700, Apr. 2019.

[20] Y. Yan, D. Shi, D. Bian, B. Huang, Z. Yi, and Z. Wang, "Small-signal stability analysis and performance evaluation of microgrids under distributed control," IEEE Trans. Smart Grid, vol. 10, no. 5, pp. 48484858, Sep. 2018.

[21] M. Raeispour, H. Atrianfar, H. R. Baghaee, and G. Gharehpetian, "Resilient $\mathrm{H} \infty$ consensus-based control of autonomous AC microgrids with uncertain time-delayed communications," IEEE Trans. Smart Grid, vol. 11, no. 5, pp. 3871-3884, May. 2020.

[22] R. Zhang and B. Hredzak, "Distributed control system with aperiodic sampled time-delayed data for batteries and renewable energy sources in microgrid," IEEE Trans. Sustain. Energy, vol. 11, no. 2, pp. 1013-1022, May. 2019.

[23] G. Lou, W. Gu, X. Lu, Y. Xu, and H. Hong, "Distributed secondary voltage control in islanded microgrids with consideration of communication network and time delays," IEEE Trans. Smart Grid, vol. 11, no. 5, pp. 3702-3715, Mar. 2020.

[24] C. Ahumada, R. Cárdenas, D. Saez, and J. M. Guerrero, "Secondary control strategies for frequency restoration in islanded microgrids with consideration of communication delays," IEEE Trans. Smart Grid, vol. 7, no. 3, pp. 1430-1441, Aug. 2015. 
[25] Z. Lian, et al., "Distributed event-triggered control for frequency restoration and active power allocation in microgrids with varying communication time delays," IEEE Trans. Ind. Electron., Aug. 2020.

[26] H. Li, Z. Sun, M.-Y. Chow, and F. Sun, "Gain-scheduling-based state feedback integral control for networked control systems," IEEE Trans. Ind. Electron., vol. 58, no. 6, pp. 2465-2472, Aug. 2010.

[27] N. Vafamand, M. H. Khooban, T. Dragičević, J. Boudjadar, and M. H. Asemani, "Time-delayed stabilizing secondary load frequency control of shipboard microgrids," IEEE Systems Journal, vol. 13, no. 3, pp. 32333241, Feb. 2019.

[28] X. Wu et al., "Delay-dependent small-signal stability analysis and compensation method for distributed secondary control of microgrids," IEEE Access, vol. 7, pp. 170919-170935, Nov. 2019.

[29] W. Yao, Y. Wang, Y. Xu, and R. Naayagi, "Communication Time-Delay Stability Margin Analysis of the Islanded Microgrid under Distributed Secondary Control," in 2020 IEEE Power \& Energy Society General Meeting (PESGM), Montreal, QC, Canada, 2020.

[30] J. W. Stahlhut, et al., "Latency viewed as a stochastic process and its impact on wide area power system control signals," IEEE Trans. Power Syst., vol. 23, no. 1, pp. 84-91, Feb. 2008.

[31] A. G. MacFarlane and I. Postlethwaite, "The generalized Nyquist stability criterion and multivariable root loci," Int. J. of Control, vol. 25, no. 1, pp. 81-127, Jun. 1976.

[32] Y. He, M. Wu, J.-H. She, and G.-P. Liu, "Delay-dependent robust stability criteria for uncertain neutral systems with mixed delays," Syst. Control Lett., vol. 51, no. 1, pp. 57-65, Jan. 2004.

[33] J. H. Park, "Stability criterion for neutral differential systems with mixed multiple time-varying delay arguments," Mathematics and Computers in Simulation, vol. 59, no. 5, pp. 401-412, Jun. 2002.

[34] The Renewable Energy Integration Demonstrator-Singapore REIDS [Online].Available:http://erian.ntu.edu.sg/REIDS/Pages/AboutREIDS.a spx

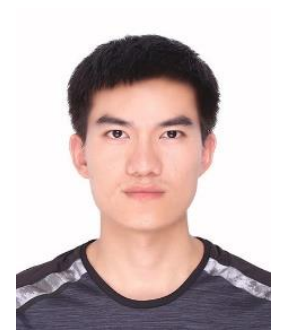

Weitao Yao (S'18) received the B.S. degree in Electrical Engineering and Automation from South China University of Technology, Guangzhou China in 2015, and the M.Sc. degree in Power Engineering from Nanyang Technological University, Singapore in 2018. He is currently working toward the Ph.D degree at ERI@N, Interdisciplinary Graduated School, Nanyang Technological University. His research interests include time-delayed stability analysis and distributed control for microgrid.

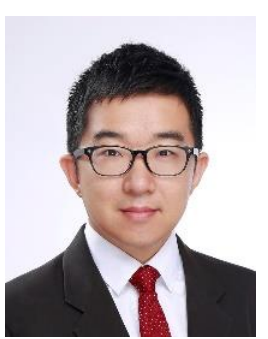

Yu Wang (S'12-M'17) received the B.Eng. degree in Electrical Engineering and Automation from Wuhan University, Wuhan China in 2011, and the M.Sc. and $\mathrm{Ph} . \mathrm{D}$. degree in Power Engineering from Nanyang Technological University, Singapore in 2012 and 2017 , respectively. He is currently a research scientist in Rolls-Royce@ NTU Corporate Lab, Singapore. His research interests include control and optimization for power systems and microgrids.

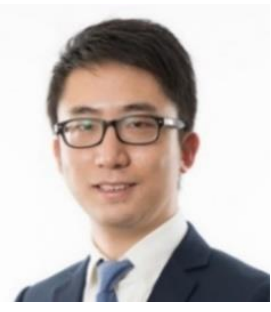

Yan Xu (S'10-M'13-SM'19) received the B.E. and M.E degrees from South China University of Technology, Guangzhou, China in 2008 and 2011, respectively, and the Ph.D. degree from The University of Newcastle, Australia, in 2013. He conducted postdoctoral research with the University of Sydney Postdoctoral Fellowship, and then joined Nanyang Technological University (NTU) with The Nanyang Assistant Professorship. $\mathrm{He}$ is now an Associate Professor at School of Electrical and Electronic Engineering and a Cluster Director at Energy Research Institute @ NTU (ERI@N). His research interests include power system stability and control, microgrid, and data-analytics for smart grid applications. Dr. Xu is an Editor for IEEE Transactions (TSG and TPWRS), IET Journals (GTD and ECE), and China's power engineering international journals (CSEE JPES and MPCE). He is also serving as the Chairman for IEEE Power \& Energy Society Singapore Chapter.

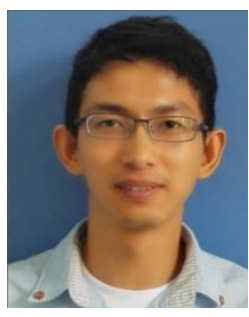

Chao Deng received the Ph.D. degree in control engineering from Northeastern University, China, in 2018. From May 2018 to May 2021, he was a Research Fellow at the School of Electrical and Electronic Engineering in Nanyang Technological University, Singapore. Currently, he is currently a faculty at the Institute of Advanced Technology, Nanjing University of Posts and Telecommunications, China.

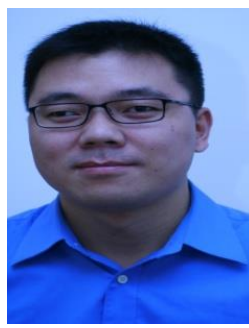

Qiuwei Wu (M'08-SM'15) obtained the PhD degree in Power System Engineering from Nanyang Technological University, Singapore, in 2009. He is an Associate Professor with the Department of Electrical Engineering, Technical University of Denmark (DTU)). His research interests are operation and control of power systems with high penetration of renewables, including wind power modelling and control, active distribution networks, and operation of integrated energy systems. 